REVI EWS

\title{
An update on the management of systemic lupus erythematosus
}

\author{
Ragan Sweet ${ }^{1}$, Soheyla Mahdavian ${ }^{1}$, Angela Singh ${ }^{1}$, Patty Ghazvini ${ }^{1}$, Ta'Shae McKinnon ${ }^{1}$, Deidra \\ J ones $^{2}$
}

1. Florida A\&M University College of Pharmacy and Pharmaceutical Sciences, Tallahassee, FL, U.S.A. 2. St. Vincent's Southside Hospital, Jacksonville, FL, U.S.A.

Correspondence: Soheyla Mahdavian. Address: Florida A\&M University College of Pharmacy and Pharmaceutical Sciences, Tallahassee, FI, U.S.A. Email: soheyla.mahdavian@famu.edu

Received: March 22, 2013

DOI : $10.5430 /$ jhm.v3n2p16

Accepted: July 21, 2013

Online Published: October 8, 2013

\section{Abstract}

Systemic lupus erythematosus (SLE) is an autoimmune disease that affecting at least 1.5 million Americans. The exact cause of SLE is unknown; however there are numerous factors that are thought to play a role in the development of SLE. While there are several medications available for the management of SLE, Belimumab is the first medication approved for SLE management in over 50 years. Researchers are investigating other medications that may provide some benefit in SLE management and improve patient outcomes. These emerging treatments explore other mechanisms for management and targeting symptoms. SLE has a higher mortality rate than other rheumatic diseases and new and continuous research is needed with the aim to decrease the number of deaths and improve patients' quality of life.

\section{Key words}

SLE, Autoimmune, Lupus, Pharmacotherapy, Interferon

\section{Background}

Systemic lupus erythematosus (SLE) is an autoimmune disease that has the potential to affect every organ in the body; however the disease affects most commonly the skin, kidneys, brain, and joints. According to the Lupus Foundation of America, at least 1.5 million Americans have SLE, although the actual number may be higher; there have been no large-scale studies to solidify the exact number of individuals living with SLE in the U.S. Annually, there are more than 16,000 new cases of SLE reported across the country. Women of childbearing age (15-44) are considered the population mostly affected by Lupus. Although people of all races and ethnic groups can develop lupus, African-American women are 2-3 times more likely to develop SLE than any other population. Over the years, the life expectancy of such patients has improved from an approximate 4 -year survival rate of $50 \%$ in the 1950 s to a 15 year survival rate of $80 \%$ today. The leading cause of death in patients with SLE is kidney failure; however other causes include infections, cerebrovascular disease, cardiovascular disease, and cancer ${ }^{[1]}$.

The exact cause of SLE is not known, however environmental, genetic, and hormonal factors are all thought to possibly lead to the development and expression of SLE. Current data illustrates the risk for development of SLE in siblings of individuals with the disease is about 20 times higher than that of the general population. The rate of identical twins 
developing SLE ranges from 24\%-58\%, whereas with non-identical twins the rate is 3\%-10\%. There have been at least 100 genes linked to SLE in humans but based upon evidence only a few genes may actually be significant. Various environmental agents that may prompt or exacerbate SLE include drugs, such as procainamide, isoniazid, methyldopa and hydralazine, ultraviolet rays from sun and fluorescent light bulbs, an infection, exhaustion, a cold or a viral illness, an injury and emotional stress such as divorce. Estrogen has also been found to play an important role in SLE. When estrogen production is high, women tend to have more SLE symptoms before menstrual periods and/or pregnancy. This may indicate that there is a correlation between the amount of estrogen and the severity of SLE but it does not demonstrate that estrogen or any other hormone causes lupus.

SLE is one of the most diverse autoimmune diseases as it may affect any organ in the body and display a broad spectrum of clinical and immunological manifestations. The most significant event in the progression of SLE is excessive and abnormal autoantibody production and the formation of immune complexes. In patients with SLE, B cell activation is abnormal and $\mathrm{T}$ cell function displays abnormalities. Numerous mechanisms lead to B-cell hyperactivity, including loss of immune self-tolerance and high antigenic load consisting of environmental and self-antigens presented to B cells by other $\mathrm{B}$ cells or specific antigen-presenting cells. There is a move of the $\mathrm{T}$ cell function towards $\mathrm{B}$ cell function leading to intensified autoantibody production. The rationale for the defective TH1 responses in SLE still remain inconclusive but possible mechanisms are down-regulation by excessive Th2 cytokines, defective interaction between APCs and T cells, the suppressive effects of CD8+ T cells and natural killer (NK) cells, the presence of IL-2 inhibitors, and the down-regulation of IL-2 receptors ${ }^{[2,3]}$.

Multiple types of autoantibodies are set to inactivate the nuclear constituents of the cell; these are known as antinuclear antibodies. Some of these antinuclear antibodies are essential in diagnosing SLE and may present in different tissues in the body. These antibodies attack various components of the cells such as DNA and are generally present many years before SLE is detected. Defective immune regulation leads to B-cell hyperactivity and excessive production of pathogenic autoantibodies with defective clearance of apoptotic cells. Meanwhile, the development and deposition of immune complexes in association with complement activation and faulty clearance of these structures result in tissue injury and gradual loss of organ function.

Patients with SLE can present in a variety of ways. All signs and symptoms may or may not be present in every patient. There are many steps involved in order for a patient to be diagnosed with SLE. First, a patient must present with at least four of the diagnostic criteria found in Table 1. These diagnostic criteria, however, do not have to appear simultaneously in order for SLE to be suspected.

In order to conclude a diagnosis of SLE, a series of blood tests are normally conducted. These tests may include antinuclear antibody titer, C-reactive protein, or erythrocyte sedimentation rate, all of which are not specific for SLE but abnormal results can consolidate a suspected diagnosis. Erythrocyte sedimentation rate (ESR) and C-reactive protein (CRP) may be ordered and are increased in the presence of inflammation. Anti-nuclear antibody (ANA) titers reveal if autoantibodies are present; however this does not confirm a diagnosis of SLE as this titer may be positive in other autoimmune diseases. Blood tests more specific to SLE include the anti-DNA titer and anti-Sm titer.

After an SLE diagnosis, a patient is started on medications to resolve current symptoms and prevent flare-ups of the disease. A patient experiencing a flare-up could present one the following symptoms:

- Loss of appetite, nausea, or vomiting

- Generalized achiness

- Increased hair loss

- Fever 
- Recurring nose bleeds

- Painful or swollen joints

- Returning rash or sores

These flare-ups are normally managed with corticosteroids until symptoms are put into remission again and medications may be adjusted.

\section{Management of SLE}

There are many different treatment options for SLE. Drug therapy is driven by pattern of disease and the severity of organ involvement. This section of the article will aim to discuss pharmacological treatment of SLE. The goal of treatment in patients with SLE is to moderate the immune system. There are several pharmacological options available for SLE and their potential promises to improve patient outcomes have been shown. Treatment is highly individualized and based on organ systems involved. Off-label uses of medications are often relied upon by healthcare providers ${ }^{[4]}$.

There are non-pharmacological therapies that will help in the management of SLE. Patients should avoid direct exposure to sunlight and use protective clothing. Sunscreens, SPF 55 or greater are essential to protect against both ultraviolet A and ultraviolet B rays. Other exacerbating factors, such as tanning beds and smoking, are best avoided by the patient. It is imperative the adequate intake of vitamin $\mathrm{D}$ and calcium especially for those patients with long-term glucocorticoid therapy ${ }^{[5]}$.

Pharmacological treatment of SLE is driven by clinical manifestation and organ involvement. Ninety percent of patients with SLE experience arthralgia and polyarthritis ${ }^{[3]}$. NSAIDs are often used first and work by inhibiting cyclooxygenase-2 (COX-2), which inhibits the production of prostaglandins which mediate inflammation and pain. Along with the inhibition of COX-2 comes inhibition of COX-1, which inhibits the production of prostaglandins. Inhibition of prostaglandins, which protects the lining of the gastrointestinal tract, causes concern for potential gastrointestinal bleeding. Therefore there is a long term risk associated with long term use of NSAIDs. NSAIDs with higher COX-2 selectivity are favored for long term use, and patients may require the addition of a proton pump inhibitor or histamine-2 receptor blocker. Celecoxib (Celebrex) is a COX-2 inhibitor, 100-200 mg/daily, may be used with caution as the sulfa component may exacerbate symptoms of SLE. Other NSAIDs that are generally used include ibuprofen $800 \mathrm{mg}$ four times a day, naproxen $500 \mathrm{mg}$ daily, or nabumetone 1 gram twice daily.

For those not responding to NSAIDs or who are experiencing flares, antimalarials may be added to therapy. The mechanism of action of antimalarial medications is not fully understood, but they have been beneficial in the treatment of flare ups and cutaneous manifestations. They have a beneficial effect of stabilizing cell membranes and warding off complications of long-term inflammation in blood vessels. They also play a role in inhibiting proteins called Toll-Like Receptors, which play a role in the initial body defense against microbes. Toll-Like Receptors contribute to autoimmunity by triggering the production of interferon alpha response in circulating genetic material and autoantibodies. Their impact on immune suppression is relatively mild and they are relatively safe. Their ability to block UV light absorption helps protect against cutaneous lesions. Antimalarials do not generally work quickly and may take up to several months to reach their full effectiveness. When flares are moderate to severe antimalarials are often paired with stronger immune modulators or steroids. Long-term studies of the use of antimalarials have also shown an added benefit in the prevention of major damage to the kidneys and central nervous system. Hydroxychloroquine is the most frequently prescribed of all the antimalarials. The usual dose is $200 \mathrm{mg} /$ day or $200 \mathrm{mg}$ twice a day. Weight should be considered when dosing hydroxychloroquine to avoid many side effects. Doses should remain at or below $6.5 \mathrm{mg} / \mathrm{kg}$ body weight. Common side effects of hydroxychloroquine are headache, lightheadedness, dermatological reactions, and gastrointestinal upset. A major potential, but rare, side effect of hydroxychloroquine use is possible damage to the retina at the back of the eye, 
which is dose related. Low doses currently used in the treatment of SLE are rarely associated with retinal damage. Patients must see an eye doctor or ophthalmologist prior to beginning treatment with hydroxychloroquine in order to have a baseline examination. Follow-up examinations must occur every three to six months thereafter. Other rare side effects include cardiac myopathy, convulsions, skeletal myopathy, toxic neuropathy, and convulsions ${ }^{[6]}$.

Methotrexate is used for resistant inflammatory arthritis and can reduce the dose of glucocorticoids. It is a folate antagonist that exhibits immunosuppressive and anti-inflammatory effects. Methotrexate is also effective in treating other clinical manifestation of SLE such as skin rashes, and may allow for reduction in corticosteroid dosing. Methotrexate may be initiated at $7.5 \mathrm{mg} /$ week and increased monthly as tolerated. A 6 month double-blind placebo-controlled RCT performed by Carneiro and Sato showed improvement in management of joint pain with the use of methotrexate. Patients used methotrexate $15-20 \mathrm{mg}$ weekly; at the end of the study 16 of 19 completers in the placebo group compared with 1 of 18 in the methotrexate group had joint complaints $(P<0.001)$. In addition to decreased joint pain, 13 patients in the methotrexate group required a lower dose of glucocorticoids compared to 1 in the placebo group. Toxicity risks of methotrexate are greater in SLE patients than that in RA patients. Toxicity risks of methotrexate increases in patient with renal insufficiency namely with a serum creatinine above two. Liver dysfunction and pneumonitis are occasional adverse effects seen with the use of methotrexate. Patients' blood counts and liver enzymes must regularly be monitored and dosages should be modified if side effects are noted.

Patients presenting with more significant organ involvement such as lupus nephritis require induction therapy. Induction therapy is given to produce remission of active disease. Kidney Disease: Improving Global Outcomes clinical guidelines and the joint European League against Rheumatism and European Association-European Dialysis and Transplant Association guidelines both state induction therapy should include glucocorticoids combined with cyclophosphamide or mycophenolate mofetil (MMF) for patient with Class III/IV. Methylprednisolone 500-1000 mg IV for 3 days is given to induce a rapid immunosuppressive effect with cyclophosphamide or MMF. Prednisone $0.5-1 \mathrm{mg} / \mathrm{kg}$ per day tapered after a few weeks to the lowest effective dose for maintenance. MMF is preferred to cyclophosphamide in African Americans and Hispanics ${ }^{[4]}$. MMF 2-3 grams a day is given for 6 months and then decreased to 1-2 grams/day or azathioprine 2 $\mathrm{mg} / \mathrm{kg} /$ day with or without a low daily dose of glucocorticoid. In case of no improvement after 6 months of cyclophosphamide with pulsed glucocorticoid the option is daily glucocorticoid administration. If the patient appears to improve, MMF 1-2 gram/day or azathioprine $2 \mathrm{mg} / \mathrm{kg} / \mathrm{day}$ with or without a low dose glucocorticoid may be utilized ${ }^{[7]}$. If there is no improvement the patient may require rituximab, which will be discussed later, with a glucocorticoid. Alternatively patient may receive cyclophosphamide high dose or low dose as induction therapy. Low dose cyclophosphamide is $500 \mathrm{mg}$ IV every 2 weeks for 6 weeks followed up with MMF or azathioprine. High dose cyclophosphamide is $500-1,000 \mathrm{mg} / \mathrm{m}^{2}$ IV every month for 6 months. For patients presenting no improvement, it is recommended MMF 2-3 grams daily for 6 months with pulse glucocorticoid followed by daily glucocorticoids. The improvement after this therapy should be followed by MMF or azathioprine maintenance dosing. If they haven't improved then the patient may try rituximab with a glucocorticoid. For those with class V, MMF 2-3 gram daily for 6 months with prednisone $0.5 \mathrm{mg} / \mathrm{kg} /$ day for 6 months followed by MMF or azathioprine maintenance dosing for those who have improved. Those who have not improved should receive cyclophosphamide $5-1,000 \mathrm{mg} / \mathrm{m}^{2}$ monthly for 6 months plus glucocorticoid pulse then prednisone $0.5-1.0 \mathrm{mg} / \mathrm{kg} / \mathrm{day}{ }^{[7]}$.

Azathioprine is a pro-drug that is metabolized to 6-mercaptopurine. It ultimately reduces the amount of circulating B and T lymphocytes, immunoglobulin synthesis, and diminished interleukin-2 secretion. Adverse effects of azathioprine include gastrointestinal upset, infection, and bone marrow suppression. Patients' $\mathrm{CBC}$ should be monitored every two weeks then every four to six weeks, and dose reduced for patients who develop leukopenia ( $\mathrm{WBC}<4,000 / \mathrm{mm}^{3}$ ) or thrombocytopenia (platelet $<150,000 / \mathrm{mm}^{3}$ ) ${ }^{[8]}$. Cyclophosphamide is an alkylating agent that is a very potent immunosuppressive therapy. Those on cyclophosphamide should have a $\mathrm{CBC}$ to monitor for leukopenia, $\mathrm{SCr}$, to monitor for renal function and need for dose adjustment while on therapy. A urinalysis monthly while on therapy and a year thereafter should be obtained to 
monitor for drug-induced cystitis. LFTs should be drawn with other blood markers to monitor for drug-induced hepatitis ${ }^{[9]}$.

Belimumab is a newer therapy whose role in SLE has not been fully defined. Belimumab is a human monoclonal antibody that blocks the biological activity of B-lymphocyte stimulator or BLyS, which is a naturally occurring protein discovered at the Human Genome Sciences. BLyS prolongs the survival of B cells which contribute to the production of antibodies. Belimumab can reduce autoantibody levels and help control autoimmune disease activity and is indicated for patients with active, autoantibody-positive, SLE who are receiving standard therapy ${ }^{[4]}$. It however has not been adequately studied in patients with severe active SLE nephritis or CNS SLE nor those who previously received rituximab or cyclophosphamide. Two trials BLISS-52 and BLISS-76 comparing belimumab $1 \mathrm{mg} / \mathrm{kg}$ and $10 \mathrm{mg} / \mathrm{kg}$ to placebo had as a primary endpoint an improvement of SRI rates. BLISS-52 showed statistical significance when compared to placebo, $1 \mathrm{mg} / \mathrm{kg}(p<0.006)$ and $10 \mathrm{mg} / \mathrm{kg}(p<0.006)^{[11]}$. Recommended dose regimen is $10 \mathrm{mg} / \mathrm{kg}$ every 2 weeks for three doses then every 4 weeks thereafter. The most common adverse reactions are nausea, diarrhea, fever, inflammation of the nose and throat, bronchitis, insomnia, pain in extremity, depression, and migraine. Until further studies are conducted to define the clinical utility of belimumab its use should be limited to patients with musculoskeletal disease or cutaneous disease ${ }^{[5]}$.

\section{New and emerging treatments}

There are several medications undergoing investigation to determine their role in the current management of systemic SLE. The classes of medications and/or procedures include immunosuppressives (leflunomide, mycophenolate) monoclonal antibodies (rituximab, epratuzumab), stem cell transplantation, biopharmaceuticals (atacicept, abatacept, blisibimod), and others.

The immunosuppressive agents are normally used to decrease the risk of rejection in transplant organs. In SLE, each immunosuppressive medication has a different mechanism of action; however in general, they work by decreasing or preventing immune responses. The immunosuppressives currently being tested for efficacy in SLE include leflunomide (Arava $\left.{ }^{\circledR}\right)$ and mycophenolate $\left(\right.$ CellCept $\left.{ }^{\circledR}\right)$.

Leflunomide may be used as an alternative to methotrexate in patients who cannot tolerate it. Leflunomide, an isoxazide derivative, inhibits the synthesis of ribonucleotide uridine monophosphate pyrimidine. It is currently FDA approved for the management of rheumatoid arthritis. Loading doses are not required and associated with higher side effects including diarrhea and other gastrointestinal disturbances. Recommended starting dose is $20 \mathrm{mg} / \mathrm{day}$. Leflunomide has been studied in both open-label and double-blind trials. In a study conducted by Suess and Sticherling, leflunomide produced conflicting results when used for a flare-up with cutaneous involvement. Half of the participants reported improvement in symptoms, while the other half noted no changes; the results were not statistically significant and provided no definitive conclusion on its efficacy. While some patients noted improvement in symptoms, leflunomide can actually worsen SLE and should therefore be used with caution. It is thought that the suppression of tumor necrosis factor $\alpha$ associated with leflunomide may be the mechanism behind the reactivation of SLE ${ }^{[12]}$.

Mycophenolate mofetil is an alternative to cyclophosphamide as initial therapy after induction therapy. This drug target is white blood cells responsible for initiation and progression of the inflammatory response and tissue damage in the joints, skin, and kidneys of people with lupus. Mycophenolate mofetil (CellCept) is initiated at $500 \mathrm{mg}$ twice daily and titrated up by $500 \mathrm{mg} /$ dose weekly to a goal of $1500 \mathrm{mg}$ twice daily by week three. It is currently used in the management of lupus nephritis and transplant rejection prophylaxis ${ }^{[13]}$.

Monoclonal antibody therapy targets both $\mathrm{B}$ and $\mathrm{T}$ lymphocytes, which are the white blood cells associated with autoantibody production in lupus. Rituximab is a monoclonal anti-B lymphocyte antibody that decreases the abnormal B-cell activation occurring in SLE. It may be beneficial for patients that have failed other therapy. Rituximab combined 
with cyclophosphamide and high dose glucocorticoids has shown promise in observational studies. However, case reports of progressive multifocal leukoencephalopathy (PML) after the rituximab administration (chimeric anti-CD20 monoclonal agents) has prompted the FDA to issue an alert to healthcare provider. The majority of PML cases associated with rituximab was observed in patients with additional immunosuppressive therapy such as chemotherapy, cyclophosphamide, and others. In any case, the continuous monitoring is recommended. Leonardo and colleagues have studied rituximab in Phase I and II trials for the treatment of refractory SLE. According to an open-label study conducted by the same investigators, rituximab led to clinical remission for an average of 12.6 months and a significant depletion in B-cells was observed in the majority of patients ${ }^{[14,15]}$.

Epratuzumab is a humanized monoclonal antibody binding to CD22 on B cell surfaces. EMBLEM in phase II for dose adjusting and safety, as well as EMBODY, a phase III trial, are currently in progress and have shown to be promising. Available data indicate that epratuzumab and Lupuzor ${ }^{\circledR}$ could be more beneficial than belimumab ${ }^{[14,16]}$.

Lupuzor is a peptide designed to inhibit $\mathrm{T}$ cell reactivity with major histocompatibility complex (MHC)-presenting self-peptides. In an open label study of patients with moderately active SLE who were receiving a small dose of Lupuzor, significant improvement was observed in both the physician's global assessment and SLE activity index. Lupuzor was generally well tolerated in this open-label trial ${ }^{[16]}$.

CNTO 136 (sirukumab) is an intravenous human anti-IL 6 that is currently undergoing Phase I testing for safety and pharmacokinetics. There are currently no results available ${ }^{[17]}$.

Hematopoietic and autologous stem cell transplant are currently in clinical trials for severe lupus. Stem cell transplantation is proposed to provide a memory $\mathrm{T}$ cell influenced free period, during which maturation of new lymphocyte progenitors can occur without the interference of anti-self activity. Stem cell transplantation is a high risk procedure and can be effective in controlling the symptoms of SLE however some could experience a recurrence of their SLE over time ${ }^{[18]}$.

Biologics are genetically engineered to interfere with the action of cells involved in the immune response. Atacicept is a recombinant fusion protein. It targets B lymphocyte stimulator or BLyS and A proliferation-inducing ligand or APRIL. BLyS and APRIL are B-cells activating factors of the tumor necrosis family. Clinical studies conducted by Dall'Era and colleagues show a dose-dependent decrease in peripheral blood lymphocytes and immunoglobulin levels. However study of atacicpet in patients with SLE nephritis was halted secondary to increased incidence of infection when used concurrently with mycophenolate. Studies are continuing in non-nephritis patients.

Abatacept blocks T cell activation and although early studies of abatacept did not show improvement of cardiovascular, musculoskeletal or cutaneous SLE, new studies are ongoing to adjust variables. In early studies both arms of the protocol received high-dose steroids for three months, and flares did not differ in part because abatacept takes six months to become optimally effective. Studies conducted by Merrill and colleagues concluded that abatacept was not more effective than placebo at reducing SLE flares.

Blisibimod is a selective peptibody antagonist of the B cell activating factor (BAFF) cytokine. The BAFF cytokine is a member of the tumor necrosis family that plays a critical role in the development, maintenance, and survival of B-cells. Blisibimod is currently in Phase III studies for patients who have not obtained optimal SLE resolution with corticosteroid therapy. The compound has shown statistically significant responses in a 24-week study $(p<0.001)$. It can be administered either subcutaneously or intravenously and has exhibited similar pharmacokinetics for both routes. Compared with other BAFF antagonists, Blisibimod offers flexible dosing with both cited routes, selective modulation and reduction of B-cell, ability to bind both membrane-bound and soluble BAFF, and structural differences that alter the pharmacokinetics and pharmacodynamics ${ }^{[19,20]}$. 


\section{Conclusion}

SLE is one of the most diverse autoimmune diseases with many different immunological manifestations. Therefore, it is essential the continuous research and development of new therapies. Pharmacotherapy of SLE is highly individualized, and involves tailoring treatment to the patients' specific needs. Non-pharmacotherapy such as protecting against ultraviolet rays and adequate vitamin supplementation can be instituted in addition to drug therapy. Treatment options like NSAID, methotrexate, azathioprine, and belimumab are currently available to patients, whereas newer agents are undergoing investigation for SLE. The new and emerging treatments present various mechanisms, mostly targeting B cell activation - the major aspect of the progression of SLE - in order to manage the disease at all levels. Monoclonal antibodies are a fast growing group of compounds used to treat many autoimmune conditions including SLE. A further understanding of the disease processes will lead to advances in the development of new therapies.

\section{References}

[1] Ward M, Pyun E, Studenski S. Causes of death in systemic lupus erythematosus. Long-term followup of an inception cohort. Arthritis Rheum. 1995; 38(10): 1492-1499. PMid:7575699 http://dx.doi.org/10.1002/art.1780381016

[2] Mok CC, Lau CS. Pathogenesis of systemic lupus erythematosus. Journal of Clinical Pathology. 2003; 56: $481-490$. http://dx.doi.org/10.1136/jcp.56.7.481

[3] Rhaman A, Isenberg D. Systemic Lupus Erythematosus. New England Journal of Medicine. 2008; 358(9): $929-939$. PMid:18305268 http://dx.doi.org/10.1056/NEJMra071297

[4] Management of Systemic Lupus Erythematosus. Pharmacist's Letter. 2011; 27(5): 270512.

[5] Schur PH, Wallace DJ. Overview of the therapy and prognosis of systemic lupus erythematosus in adults. Wolters, Kluwer Health Uptodate[Internet]. 2013 Mar 1[cited 2013 Mar 24]. Available from:

http://www.uptodate.com/contents/overview-of-the-therapy-and-prognosis-of-systemic-lupus-erythematosus-in-adults

[6] "Hydroxychloroquine (Plaquenil) and Systemic Lupus Erythematosus". Pharmacist's Letter. 2007; 23: 1-3

[7] Cervera R, O’Neill S. "Systemic Lupus Erythematosus”. Best Practice and Research Clinical Rhematology. 2010; $24: 841-855$. PMid:21665130 http://dx.doi.org/10.1016/j.berh.2010.10.006

[8] Hahn B, McMahon M, Wilkinson A, et al. "American College of Rheumatology Guidelines for Screening, Treatment, and Management of Lupus Nephritis” Arthritis Care and Research. 2012; 64(6): 797-808.

PMid:22556106 http://dx.doi.org/10.1002/acr.21664

[9] Belmont MH. Pharmacology and Side Effects of Azathioprine when Used in Rheumatic Disease. Wolters, Kluwer Health Uptodate [Internet]. 2010 Feb 11[cited 2013 Mar 25]. Available from:

http://www.uptodate.com/contents/pharmacology-and-side-effects-of-azathioprine-when-used-in-rheumatic-diseases

[10] Stone JH. General Principles of the use of Cyclophophamide in Rheumatic and Renal Disease. Wolters, Kluwer Health Uptodate [Internet]. 2012 Aug 31[cited 2013 Mar 25]. Available from:

www.uptotdate.com/contents/general-principles-of-the-use-of-cyclophosphamide-in-rheumatic-and-renal-disease

[11] van Vollenhoven RF, Petri MA, Cervera C, et al. Belimumab in the Treatment of Systemic Lupus Erythematosus: High Disease Activity Predictors of Response. Annals of Rheumatic Diseases. 2012: 1343-1349.

PMid:22337213 http://dx.doi.org/10.1136/annrheumdis-2011-200937

[12] Boyd A. Luflunomide in dermatology. J Am Acad Dermatol. 2012; 66: 673-679. PMid:21962758 http://dx.doi.org/10.1016/j.jaad.2011.08.025

[13] Iaccarino L, Rampudda M, Canova M, Libera S, et al. Mycophenolate mofetil: What is its place in the treatment of autoimmune rheumatic diseases? Autoimmunity Reviews. 2007; 6: 190-195. PMid:17289556 http://dx.doi.org/10.1016/j.autrev.2006.11.001

[14] Leandro M, Cambridge G, Edwards J, et al. B-cell depletion in the treatment of patients with systemic lupus erythematosus: a longitudinal analysis of 24 patients. Rheumatology. 2005; 44(12): 1542-1545. PMid:16188950

http://dx.doi.org/10.1093/rheumatology/kei080

[15] Tieng A, Peeva E. B-Cell-Directed Therapies in Systemic Lupus Erythematosus. Semin Arthritis Rheum. 2008; 38:218-227. PMid:18206214 http://dx.doi.org/10.1016/j.semarthrit.2007.11.003

[16] Chugh P. Lupus: Novel therapies in clinical development. European Journal of Internal Medicine. 2012; 23; $212-218$. PMid:22385876 http://dx.doi.org/10.1016/j.ejim.2011.11.001 
[17] Centocor Research \& Development, Inc. A Study of the Safety and Pharmacokinetics of CNTO 136 in Patients With Cutaneous Lupus Erythematosus and Systemic Lupus Erythematosus. Clinicaltrials.gov Database[Internet]. 2012 Jul 12[cited 2012 Dec 18]; Available from: http://clinicaltrials.gov/ct2/show/NCT01702740

[18] Pavletic S, Illei G. The role of immune ablation and stem cell transplantation in severe SLE. Best Practice \& Research in Clinical Rheumatology. 2005; 19(5): 839-858. PMid:16150406 http://dx.doi.org/10.1016/j.berh.2005.05.002

[19] Anthera Pharmaceuticals, Inc. BAFF Peptibody for the Treatment of Lupus. Anthera Pharmaceuticals, Inc [Internet]. 2012[cited 2012 Dec 18]. Available from: http://www.anthera.com/products_a623.asp

[20] Nery B. Anthera Announces the Advancement of Blisibimod into Phase 3 Development for Patients with Systemic Lupus Erythematosus (SLE). Anthera Pharmaceuticals, Inc [Internet]. 2012 Sept 17[cited 2012 Dec 18]; Available from: http://investor.anthera.com/releasedetail.cfm?ReleaseID=707227 